\title{
Dynamic Susceptibility Contrast-Enhanced MR Perfusion Imaging in Assessing Recurrent Glioblastoma Response to Superselective Intra-Arterial Bevacizumab Therapy
}

\author{
(D) R. Singh, (D). Kesavabhotla, DS.A. Kishore, (D). Zhou, (D)A.J. Tsiouris, DC.G. Filippi, (D).A. Boockvar, and (D). Kovanlikaya
} o- $\equiv$

\begin{abstract}
BACKGROUND AND PURPOSE: Recurrent glioblastoma currently has no established standard of care. We evaluated the response of recurrent glioblastoma to superselective intra-arterial cerebral infusion of bevacizumab by using dynamic susceptibility contrast-enhanced MR perfusion imaging. We hypothesized that treatment response would be associated with decreased relative CBV and relative CBF.

MATERIALS AND METHODS: Patients were accrued for this study from larger ongoing serial Phase I/II trials. Twenty-five patients (14 men, 11 women; median age, 55 years) were analyzed. Four distinct ROIs were chosen: 1) normal-appearing white matter on the contralateral side, 2) the location of the highest $T 1$ enhancement in the lesion (maximum enhancing), 3) the location of highest relative CBV in the lesion (maximum relative $\mathrm{CBV}$ ), and 4) nonenhancing $\mathrm{T} 2$ hyperintense signal abnormality surrounding the tumor (nonenhancing $\mathrm{T} 2$ hyperintensity).
\end{abstract}

RESULTS: There was a statistically significant median percentage change of $-32.34 \%(P=.001)$ in relative CBV in areas of maximum relative CBV following intra-arterial bevacizumab therapy. There was also a statistically significant median percentage decrease in relative CBF of $-30.67(P=.001)$ and $-27.25(P=.037)$ in areas of maximum relative CBV and maximum tumor enhancement, respectively. Last, a trend toward statistical significance for increasing relative CBV in nonenhancing T2 hyperintense areas (median percent change, 30.04; $P=.069$ ) was noted.

CONCLUSIONS: Dynamic susceptibility contrast-enhanced MR perfusion imaging demonstrated a significant decrease in tumor perfusion metrics within recurrent glioblastomas in response to superselective intra-arterial cerebral infusion of bevacizumab; however, these changes did not correlate with time to progression or overall survival.

ABBREVIATIONS: $B$ V = bevacizumab; DSC-MRP = dynamic susceptibility contrast-enhanced MR perfusion; $G B M=$ glioblastoma; max = maximum; NAWM = normal-appearing white matter; OS = overall survival; RANO = Response Assessment in Neuro-Oncology; rCBF = relative cerebral blood flow; rCBV $=$ relative cerebral blood volume; $\mathrm{SIACI}=$ superselective intra-arterial cerebral infusion; TTP $=$ time to progression

G lioblastoma (GBM) is the most common and lethal primary malignancy of the central nervous system. Despite a 3-pronged intervention consisting of surgical resection followed

Received November 9, 2015; accepted after revision March 30, 2016.

From the Departments of Neurological Surgery (R.S., Z.Z.) and Radiology (S.A.K., A.J.T., I.K.), Weill Cornell Medical College, New York, New York; Department of Neurological Surgery (K.K.), Northwestern University Feinberg School of Medicine, Chicago, Illinois; and Departments of Radiology (C.G.F.) and Neurological Surgery (J.A.B.), Lenox Hill Hospital, Hofstra-North Shore-LIJ School of Medicine,

New York, New York.

This work was partly supported by the Carolyn L. Kuckein Student Research Fellowship (R.S.), the Radiological Society of North America Research Medical Student Grant (K.K.), and National Cancer Institute grant No. CA130985 (J.A.B.).

Please address correspondence to Ilhami Kovanlikaya, MD, 51571 E St Room S-119, New York, NY 10021; e-mail: ilk2002@med.cornell.edu

-- Indicates open access to non-subscribers at www.ajnr.org

= Indicates article with supplemental on-line tables.

http://dx.doi.org/10.3174/ajnr.A4823 by radiation with both concurrent and adjuvant temozolomide chemotherapy, the 5-year overall survival rate of patients remains approximately $10 \%{ }^{1}$

While there is no established standard of care for recurrent GBM, bevacizumab (BV, Avastin) has emerged as a potential treatment option for recurrent GBM. BV is a humanized monoclonal antibody that exerts antineoplastic effects by inhibiting the angiogenic effects of vascular endothelial growth factor-A. ${ }^{2,3}$ Our group has used superselective intra-arterial cerebral infusion (SIACI) following blood-brain barrier disruption to improve BV delivery. ${ }^{4}$ Recently published studies from our group have shown promising results on the safety and efficacy of using SIACI delivery for BV. ${ }^{5,6}$

Although treatment with BV produces a dramatic decrease in MR imaging contrast enhancement, the degree to which these findings reflect actual antitumor effects remains unclear. ${ }^{7} \mathrm{BV}$ re- 
duces vessel permeability, which may contribute to changes in enhancement features and potentially confound the relationship between enhancement and tumor response. Hence, the ability of conventional MR imaging to determine tumor response, progression, and posttreatment effects is not well-established. ${ }^{8}$ Our group previously reported that ${ }^{1} \mathrm{H}-\mathrm{MR}$ spectroscopy may be a viable method to determine GBM response following SIACI BV, to overcome the limitations of conventional MR imaging. ${ }^{7}$

Here we evaluate the potential for using dynamic susceptibility contrast-enhanced MR perfusion (DSC-MRP) to determine GBM response to SIACI BV. Previous studies have highlighted the utility of using DSC-MRP in assessing tumor response, treatment effectiveness, and clinical outcomes in patients with GBM. ${ }^{9,10}$ Specifically, decreases in tumor relative CBV (rCBV) and tumor relative $\mathrm{CBF}(\mathrm{rCBF})$ are associated with favorable clinical outcome, suggesting that changes in $\mathrm{rCBV}$ and $\mathrm{rCBF}$ could serve as biomarkers for treatment response. ${ }^{9,10}$ We hypothesized that treatment response to SIACI BV is associated with decreased $\mathrm{rCBV}$ and $\mathrm{rCBF}$, which will correlate with improved survival outcomes.

\section{MATERIALS AND METHODS \\ Subjects}

Patients were accrued for this study from larger ongoing serial Phase I/II trials of SIACI BV and were retrospectively analyzed with approval from the institutional review board of Weill Cornell Medical College. Inclusion criteria for the Phase I/II SIACI BV trials were recurrent World Health Organization grade IV glioma refractory to previous combined radiation treatment and chemotherapy with temozolomide, a Karnofsky Performance Scale score of $>60$, and $<12$ doses of prior intravenous BV treatment. Poorly circumscribed enhancing tumors, multifocal tumors, or leptomeningeal spread of tumors were not exclusion criteria. Recurrent GBM was diagnosed by using follow-up MR imaging, Response Assessment in Neuro-Oncology (RANO) criteria for progression, ${ }^{11}$ and clinical evaluation. Patients with the following were diagnosed with recurrent disease: 1) an increase in a contrast-enhancing lesion; 2) an increase in a nonenhancing T2/ FLAIR lesion in 1 or 2 follow-up scans, which showed mass effect, infiltration of the cortical ribbon, or lesion location outside the radiation field; 3) any new lesions; or 4) clinical deterioration diagnosed with recurrent disease. Follow-up MR imaging was compared with MR imaging obtained within 48 hours after the operation to appropriately differentiate tumor recurrence from postoperative changes.

Inclusion criteria for the current study were patients from the above Phase I/II trials who underwent brain DSC-MRP imaging within 1-10 days before and 3-5 weeks after SIACI BV. Twentyfive patients ( 14 men, 11 women; median age, 55 years; range, 29-81 years) met the inclusion criteria (On-line Table 1). Seven of the 25 patients $(28 \%$ ) received intravenous BV before SIACI BV for a mean of 4.7 cycles (range, 0.5-9 cycles). All except 2 patients received steroids. Time to progression (TTP) and overall survival (OS) were calculated by using the date of the operation for primary GBM to the date of radiologic progression of disease after SIACI BV and date of death. The date of radiologic progression was determined by using strict RANO criteria by a boardcertified diagnostic radiologist with a Certificate of Added Qual- ification in neuroradiology (A.J.T., 11 years of experience) and a trained senior neuroradiologist (I.K., 20 years of subspecialty experience). ${ }^{11}$

\section{Treatment Protocol}

We have previously described the technical specifications of SIACI and BV treatment. ${ }^{4-6,12}$ Briefly, 25\% mannitol (1.4 mol/L) was infused at $10 \mathrm{~mL} / 120$ seconds to facilitate transient BBB disruption followed by SIACI BV. Subsequently, the appropriate dose of BV was infused during 15 minutes. However, because the Phase I trial aimed to determine the maximum tolerated dose of SIACI BV with analysis of 10 escalating doses $(2,4,6,8,10,11,12$, 13,14 , and $15 \mathrm{mg} / \mathrm{kg}$ ), the administered dose varied among patients selected for this study. The mean SIACI BV dose received was $12.4 \mathrm{mg} / \mathrm{kg}$ (range, $4-15 \mathrm{mg} / \mathrm{kg}$ ), with 15 patients (60\%) receiving the maximum dose of $15 \mathrm{mg} / \mathrm{kg}$. After a mean of $27 \pm 5$ days of observation, all included patients underwent postinfusion imaging. No additional therapy was initiated before the postSIACI BV MR imaging-DSC-MRP was completed. Fourteen of 25 patients $(56 \%)$ underwent various subsequent treatments after SIACI BV that included intra-arterial cetuximab, temozolomide, and/or intravenous BV. We included all imaging studies up to 6 months and then at 1 year posttreatment if available.

\section{Brain MR Imaging and DSC-MR Imaging Data Collection and Processing}

All neuroimaging examinations were conducted on a 3T HDxt 15x MR imaging scanner (GE Healthcare, Milwaukee, Wisconsin). Conventional MR imaging with a dedicated standardized SIACI BV imaging protocol (previously described) was performed. ${ }^{7}$ DSC-MR imaging data were acquired by using singleecho gradient recalled-echo echo-planar imaging, with a flip angle of $60^{\circ}$; TR/TE of 2000/20 ms; FOV of $240 \mathrm{~mm} ; 129 \times 96$; section thickness/gap of $5 \mathrm{~mm} / 0$; NEX of 1 ; number of shots of 1 . The first $0.1 \mathrm{mmol} / \mathrm{kg}$ of gadolinium administration was used as a preload for the subsequent DSC study to correct the T1weighted effects of vascular leakage on rCBV. Next, 0.10 $\mathrm{mmol} / \mathrm{kg}$ of gadolinium at $3-5 \mathrm{~mL} / \mathrm{s}$ was administered at least 5 minutes after the preload injection. ${ }^{13,14}$ The negative enhancement integration and linear fitting correction method was used for postprocessing to calculate corrected $\mathrm{rCBV}$ and rCBF. ${ }^{13}$ Functional rCBV and rCBF maps were obtained and analyzed by using Olea Sphere Version 2.3 SP2 (Olea Medical, La Ciotat, France).

\section{Selection of ROIs and Evaluation of Data}

Up to 4 distinct ROIs ranging in size from 10 to 12 voxels were chosen from the coregistered precontrast T1-weighted, postcontrast T1-weighted, and T2-FLAIR images and rCBV maps (Fig 1): 1) normal-appearing white matter (NAWM) on the contralateral side (Fig 1A), which was used to normalize rCBV and $\mathrm{rCBF}$ maps on a voxelwise basis [Normalized $\mathrm{rCBV}=$ rCBV (Lesion) / rCBV (NAWM) ]; 2) the location of highest T1 enhancement in the lesion (maximum $[\max ]$ enhancing) (Fig $1 B)$; 3 ) the location of the highest $\mathrm{rCBV}$ in the lesion ( $\max$ rCBV) (Fig 1C); and 4) nonenhancing T2 hyperintense signal abnormality surrounding the tumor (nonenhancing T2 hyper-

AJNR Am J Neuroradiol 37:1838-43 Oct 2016 www.ajnr.org 1839 


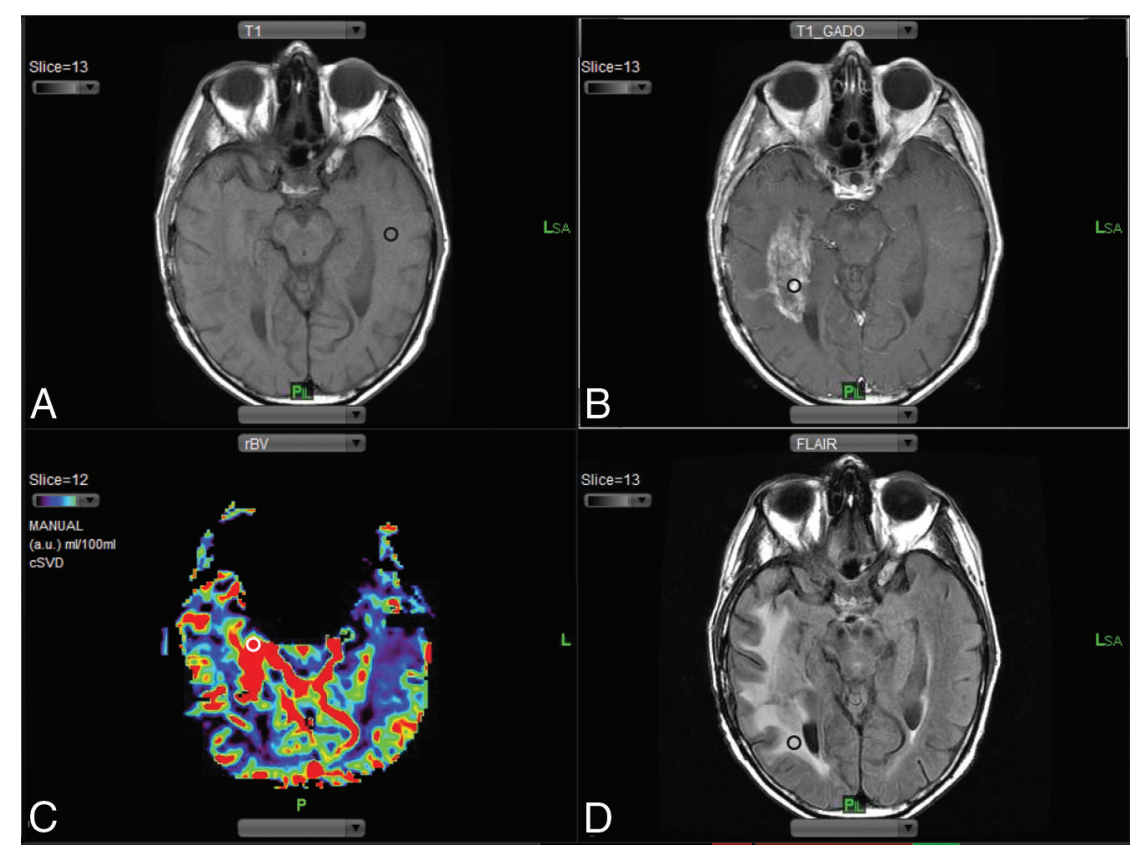

FIG 1. ROI selection. A, Precontrast TI-weighted images are used to select the ROI in the normalappearing white matter on the side contralateral to the lesion (black circle). B, Postcontrast Tl-weighted images are compared with the precontrast T1-weighted images to select the ROI representing the area of max contrast enhancement (black circle). C, Region of max rCBV is selected by using rCBV maps (white circle). D, T2-FLAIR images are used to select areas representing the nonenhancing T2 hyperintense signal abnormality surrounding the tumor (black circle).

$P=.069)$. The change in $\mathrm{rCBV}$ was not found to be statistically significant in contralateral NAWM (median percentage change, -4.255 ; range, $-82.35-143.75 ; P=.568)$. The median percentage change in $\mathrm{rCBV}$ in the nonenhancing T2 hyperintense region showed a trend toward statistically significant correlation with the presence of previous cycles of BV $(P=.062)$. Median TTP and OS were 571 and 683 days, respectively. None of the rCBV changes correlated with prolonged TTP or OS. Last, the rCBV changes were significantly different among the 4 ROIs $(P=.0003)$.

\section{Cerebral Blood Flow}

There was a statistically significant median percentage change of -30.67 (range, $-76.40-44.18 ; P=.001$ ) and -27.25 (range, $-65.99-55.60 \%$; $P=$ .037) in rCBF in areas of max rCBV and max tumor enhancement, respectively, from pre- to post-SIACI BV. The change in $\mathrm{rCBF}$ was not found to be statistically significant in contralateral NAWM (median percentage change, 0.363 ; range,

intensity) (Fig 1D). The same size and anatomically matching ROIs were manually constructed by using contrast-enhanced T1-weighted and T2-weighted images as a reference from the pre- and posttreatment MR imaging scans. Only 1 investigator (S.A.K.) placed ROIs, and all ROI placements were overseen by 2 senior investigators (A.J.T. and I.K.).

\section{Statistical Analysis}

Differences in rCBV and rCBF from pre- to post-SIACI BV (defined as median percentage change: [(Posttreatment - Pretreatment)/Pretreatment $\times 100 \%]$ ) were determined by using the Wilcoxon signed rank test. The Spearman correlation was used to assess the correlation between changes in $\mathrm{rCBV}$ and rCBF in the various ROIs and TTP and OS. Differences of rCBV and rCBF changes in ROIs were tested by using ANOVA within subjects.

\section{RESULTS}

DSC-MRP showed that SIACI BV produced changes in rCBV and rCBF (Fig 2 and On-line Table 2). Median percentage change values are reported, which were not significantly different from the mean percentage change values (On-line Table 3).

\section{Cerebral Blood Volume}

When one compared pre- and post-SIACI BV, there was a statistically significant median percentage change of -32.34 (range, $-79.18-38.90 ; P=.001$ ) in $\mathrm{rCBV}$ in areas of max rCBV. There was a trend toward statistical significance in areas of max tumor enhancement (median percentage change, -27.29; range, $-66.30-117.64 ; P=.074)$ and in nonenhancing T2 hyperintense areas (median percentage change, 30.04; range, $-83.26-255.42 \%$;
-68.77-68.95; $P=.696)$ and in the nonenhancing T2 hyperintense areas (median percentage change, 20.99; range, -63.85208.97; $P=.216$ ). None of the $\mathrm{rCBF}$ changes correlated with prolonged TTP or OS. Last, the $\mathrm{rCBF}$ changes were significantly different among the 4 ROIs $(P=.021)$.

\section{DISCUSSION}

Conventional MR imaging is currently unable to provide consistent and accurate assessment of pathology-specific tumor progression and therapeutic response, which limit its diagnostic and prognostic utility. ${ }^{8}$ This limitation has led to the development of advanced quantitative imaging techniques that provide critical information on the molecular, physiologic, and metabolic processes and properties of tumors. ${ }^{15}$ Previously, we showed that MR spectroscopic imaging, specifically choline/ $N$-acetylaspartate ratios, provided a useful tool to assess treatment response following SIACI BV. ${ }^{7}$ In the current study, we used DSC-MRP to assess GBM perfusion changes associated with SIACI BV to determine whether DSC-MRP provided useful biomarkers to determine treatment response. We also wanted to explore whether biomarkers obtained from DSC-MR imaging could reveal aspects of the complex mechanism underlying the tumoricidal effects of BV.

Antiangiogenic agents such as BV produce a marked decrease in contrast enhancement, termed "pseudoresponse," and a notable decrease in the nonenhancing T2 hyperintense areas. Standardized criteria for assessing brain tumor treatment response, including the Macdonald and the RANO criteria, fall short of definitively distinguishing tumor progression, pseudoresponse, and pseudoprogression. ${ }^{16}$ The inability of the Macdonald and RANO criteria to differentiate tumor progression, pseudore- 


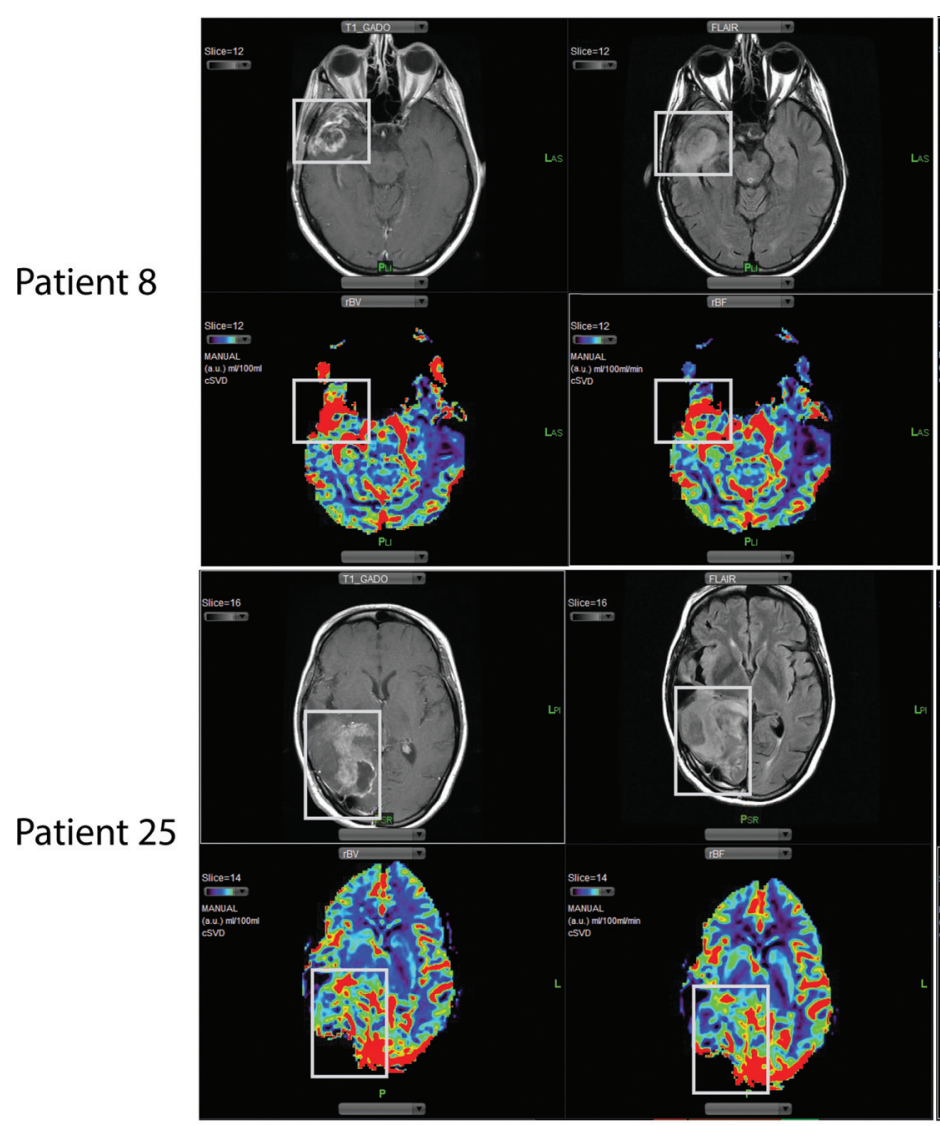

Pre-SIACI BV

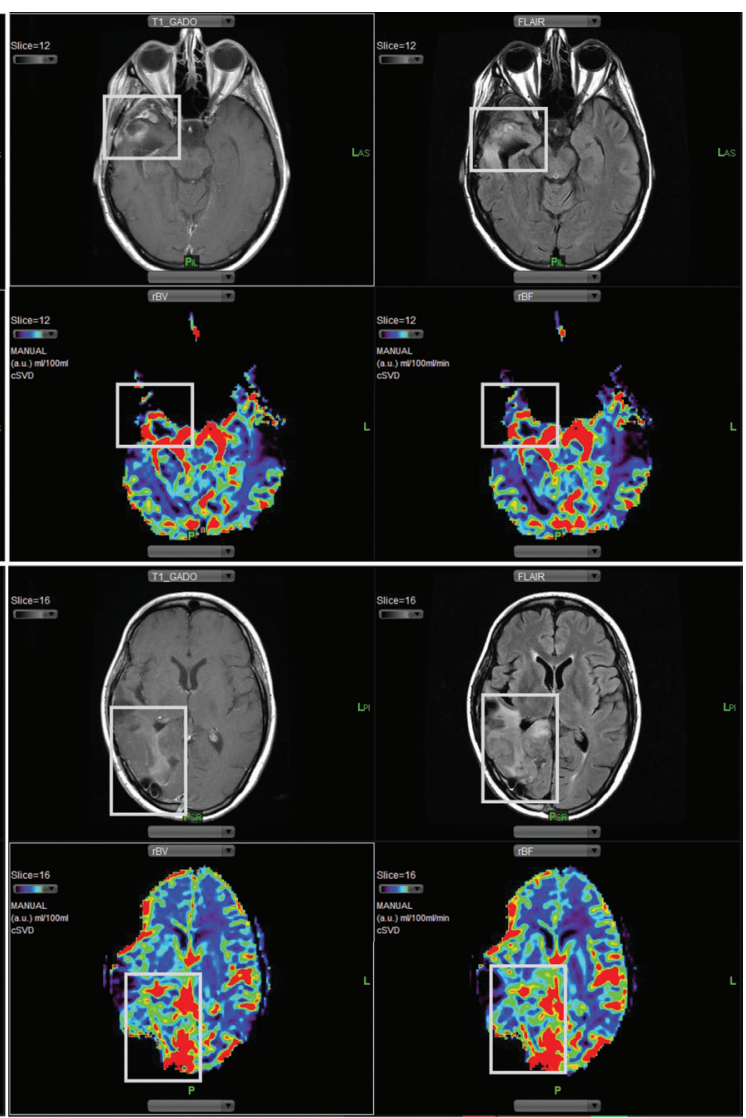

Post-SIACI BV

FIG 2. MR imaging changes from SIACI BV treatment. Imaging from 2 patients (study patients 8 and 25) demonstrates a decrease in contrast enhancement, T2 signal abnormalities, rCBV, and rCBF following SIACI BV.

sponse, and pseudoprogression is noteworthy and could lead to conflicting and confusing outcome evaluations in BV treatment. Recognition of pseudoresponse and pseudoprogression in antiangiogenic therapy is critical to appropriately determine whether the decrease in contrast enhancement reflects a true decrease in tumor burden or is simply due to normalization of BBB and tumor vasculature.

It remains unclear whether BV acts by pruning tumor vessels and killing a fraction of tumor cells; by normalizing existing tumor vasculature and the tumor microenvironment, thus increasing the delivery of chemotherapy; or by reducing the number of blood-circulating endothelial and progenitor cells, thus inhibiting neovascularization. ${ }^{17-19}$ MR diffusion-weighted, perfusionweighted, and spectroscopy imaging may provide quantitative data on the molecular and metabolic processes that underlie tumorigenesis and tumor response. MR spectroscopic imaging can be used to study neurochemical changes that may help explain the tumoricidal effects of BV. ${ }^{7}$ DSC-MRP offers another appealing parametric imaging technique to potentially elucidate the mechanism of action of BV.

DSC-MRP tracks the first pass of a bolus of gadolinium-based contrast agent through brain tissue by a series of rapid T2- or $\mathrm{T} 2^{\star}$-weighted MR images. The susceptibility effect of the paramagnetic contrast agent leads to transient decreases in $\mathrm{T} 2$ and $\mathrm{T} 2{ }^{*}$ relaxation times, resulting in signal loss in the signal intensitytime curve. The signal information can then be converted into a contrast medium concentration-time curve and used to generate parametric maps of rCBV, rCBF, and K2 (leakage coefficient). ${ }^{20,21}$ DSC-MRP is particularly sensitive to changes in tumor vasculature, which is noteworthy given that $\mathrm{BV}$ affects blood vessels. DSC-MRP, therefore, may be useful in both assessing tumor response to $\mathrm{BV}$ and better understanding the tumoricidal effects of BV.

In the present study, DSC-MRP was used to assess tumor response in 25 patients with recurrent GBM treated with SIACI BV. rCBV and rCBF were reliable biomarkers for assessing tumor response to SIACI BV. The change in rCBV from pre- to post-SIACI $\mathrm{BV}$ was statistically significant in the ROIs in max rCBV. The change in $\mathrm{rCBV}$ also showed a trend toward statistical significance in ROIs in max tumor enhancement, which was associated with an observable decrease in the contrast enhancement of the lesion. No statistically significant changes or trends were found in the contralateral NAWM. The change in $\mathrm{rCBF}$ was statistically significant in ROIs in max rCBV and max tumor enhancement, and not statistically significant in ROIs in contralateral NAWM. Collectively, these data show that the SIACI BV acted locally at the site of tumor, with minimal effect in the contralateral NAWM. A recent study reported that perfusion decreased in ipsilateral and contralateral normal-appearing brain after BV treatment. ${ }^{22}$ This study, however, obtained absolute CBV, and the route of BV administration was different from that in our study, which may explain the different findings.

AJNR Am J Neuroradiol 37:1838-43 Oct 2016 www.ajnr.org 
In our patients, SIACI BV produced a marked decrease in rCBV and $\mathrm{rCBF}$ in the max rCBV and max tumor-enhancing regions on DSC-MRP imaging. Most interesting, we also observed a trend toward statistical significance in $\mathrm{rCBV}$ increase in the nonenhancing T2 hyperintense areas surrounding the lesion. This may suggest that while the contrast-enhancing region within the tumor may reflect the treatment response to SIACI BV, it may not adequately reflect tumor burden, treatment effect, or tumor progression during or after SIACI BV treatment. It is unclear whether the increase in $\mathrm{rCBV}$ in the nonenhancing $\mathrm{T} 2$ hyperintense region reflects an increase in tumor volume or perhaps an increase in tumor invasiveness. Because several preclinical and clinical studies have reported that antiangiogenic therapy increases tumor invasiveness, ${ }^{23-25}$ the increased rCBV in the nonenhancing T2 hyperintense region approximately 1 month after SIACI BV in our study may be reflective of this phenomenon. However, increased T2 hyperintensity occurs more commonly after long-term IV BV exposure and histologically represents a low-grade infiltrative phenotype. In our study, there was no statistically significant difference in TTP and OS among patients who received intravenous BV before SIACI BV compared with those who did not. Combined radiologic and pathologic correlative studies are needed to better understand the imaging biomarkers of tumor invasiveness, especially as they pertain to antiangiogenic therapy.

Post-SIACI BV changes in MRP biomarkers did not correlate with prolonged TTP and OS. It is difficult to conclusively state whether this was due to lack of treatment effect or other confounding variables. The sample size was small and clinical heterogeneity in patients selected for inclusion in the Phase I/II SIACI BV trials should be considered. Notably, more than a quarter of our patients were exposed to BV before enrolling in SIACI BV clinical trials, and not every patient received the maximum dose of SIACI BV. Furthermore, more than half of our patients received subsequent treatment after SIACI BV, making it difficult to accurately assess the true implications of this potential treatment. Given the design, the study had limitations inherent in all retrospective reviews: Namely, our results demonstrate correlation and not causation. The subjectivity in selecting matching ROIs on pre- and posttreatment scans may have introduced sampling error. To minimize this, only one investigator (K.K.) placed ROIs, and all ROI placements were overseen by 2 senior investigators (A.J.T. and I.K.). Another limitation was that histologic specimens were not available to confirm the diagnosis of recurrent disease. While it is ideal to obtain histologic specimens of recurrent disease, it is not realistic to expect patients to agree to an additional surgical procedure for open biopsy. Furthermore, even if a biopsy is obtained, correlation with posttreatment MRP changes may not be feasible because the exact site of biopsy is often not known or identifiable after biopsy, making it difficult to correlate MRP changes with histopathologic examination. Future studies by using SIACI BV should attempt to obtain biopsy specimens of recurrent disease by using specified coordinates and match these coordinates voxel-by-voxel to post-SIACI BV treatment MRP scans.

\section{CONCLUSIONS}

This study suggests that GBM response to SIACI BV can be assessed by comparing pre- and posttreatment rCBV and rCBF changes in regions of the tumor with max $\mathrm{rCBV}$ and max enhancement. However, there was no correlation between these significant MRP biomarker changes, TTP, and OS.

Disclosures: Christopher G. Filippi-UNRELATED: Consultancy: Syntactx, Guerbet, Comments: review of brain MR images for a clinical research trial; attended Advisory Board Meeting for Guerbet in Boston as a consultant; Grants/Grants Pending: Katz Institute for Women's Health (KIWH), ${ }^{*}$ Coulter, ${ }^{*}$ Comments: KIWH grant, coinvestigator, funded from September 2015 to October 2016 for grant determining optimum imaging strategy for women with acute stroke; and Coulter grant, Principal Investigator, on the development of a novel semiautomated computer software algorithm for core infarct detection. *Money paid to the institution.

\section{REFERENCES}

1. Stupp R, Hegi ME, Mason WP, et al; European Organisation for Research and Treatment of Cancer Brain Tumour and Radiation Oncology Groups, National Cancer Institute of Canada Clinical Trials Group. Effects of radiotherapy with concomitant and adjuvant temozolomide versus radiotherapy alone on survival in glioblastoma in a randomised phase III study: 5-year analysis of the EORTCNCIC trial. Lancet Oncol 2009;10:459-66 CrossRef Medline

2. Chinot OL. Bevacizumab-based therapy in relapsed glioblastoma: rationale and clinical experience to date. Expert Rev Anticancer Ther 2012;12:1413-27 CrossRef Medline

3. Mukherji SK. Bevacizumab (Avastin). AJNR Am J Neuroradiol 2010; 31:235-36 CrossRef Medline

4. Riina HA, Fraser JF, Fralin S, et al. Superselective intraarterial cerebral infusion of bevacizumab: a revival of interventional neuro-oncology for malignant glioma. J Exp Ther Oncol 2009;8:145-50 Medline

5. Boockvar JA, Tsiouris AJ, Hofstetter CP, et al. Safety and maximum tolerated dose of superselective intraarterial cerebral infusion of bevacizumab after osmotic blood-brain barrier disruption for recurrent malignant glioma: clinical article. J Neurosurg 2011;114: 624-32 CrossRef Medline

6. Burkhardt JK, Riina H, Shin BJ, et al. Intra-arterial delivery of bevacizumab after blood-brain barrier disruption for the treatment of recurrent glioblastoma: progression-free survival and overall survival. World Neurosurg 2012;77:130-34 CrossRef Medline

7. Jeon JY, Kovanlikaya I, Boockvar JA, et al. Metabolic response of glioblastoma to superselective intra-arterial cerebral infusion of bevacizumab: a proton MR spectroscopic imaging study. AJNR Am J Neuroradiol 2012;33:2095-102 CrossRef Medline

8. Upadhyay N, Waldman AD. Conventional MRI evaluation of gliomas. Br J Radiol 2011;84(Spec No 2):S107-11 CrossRef Medline

9. Kickingereder P, Wiestler B, Burth S, et al. Relative cerebral blood volume is a potential predictive imaging biomarker of bevacizumab efficacy in recurrent glioblastoma. Neuro Oncol 2015;17: 1139-47 CrossRef Medline

10. Schmainda KM, Zhang Z, Prah M, et al. Dynamic susceptibility contrast MRI measures of relative cerebral blood volume as a prognostic marker for overall survival in recurrent glioblastoma: results from the ACRIN 6677/RTOG 0625 multicenter trial. Neuro Oncol 2015;17:1148-56 CrossRef Medline

11. Wen PY, Macdonald DR, Reardon DA, et al. Updated response assessment criteria for high-grade gliomas: response assessment in neurooncology working group. J Clin Oncol 2010;28:1963-72 CrossRef Medline

12. Shin BJ, Burkhardt JK, Riina HA, et al. Superselective intra-arterial cerebral infusion of novel agents after blood-brain disruption for the treatment of recurrent glioblastoma multiforme: a technical case series. Neurosurg Clin N Am 2012;23:323-29, ix-x CrossRef Medline

13. Boxerman JL, Schmainda KM, Weisskoff RM. Relative cerebral blood volume maps corrected for contrast agent extravasation sig- 
nificantly correlate with glioma tumor grade, whereas uncorrected maps do not. AJNR Am J Neuroradiol 2006;27:859-67 Medline

14. Paulson ES, Schmainda KM. Comparison of dynamic susceptibilityweighted contrast-enhanced MR methods: recommendations for measuring relative cerebral blood volume in brain tumors. Radiology 2008;249:601-13 CrossRef Medline

15. Kalpathy-Cramer J, Gerstner ER, Emblem KE, et al. Advanced magnetic resonance imaging of the physical processes in human glioblastoma. Cancer Res 2014;74:4622-37 CrossRef Medline

16. Huang RY, Neagu MR, Reardon DA, et al. Pitfalls in the neuroimaging of glioblastoma in the era of antiangiogenic and immuno/targeted therapy: detecting illusive disease, defining response. Front Neurol 2015;6:33 CrossRef Medline

17. Falk AT, Barrière J, François E, et al. Bevacizumab: a dose review. Crit Rev Oncol Hematol 2015;94:311-22 CrossRef Medline

18. Jain RK, Duda DG, Clark JW, et al. Lessons from phase III clinical trials on anti-VEGF therapy for cancer. Nat Clin Pract Oncol 2006;3: 24-40 CrossRef Medline

19. Okonogi N, Shirai K, Oike T, et al. Topics in chemotherapy, molecular-targeted therapy, and immunotherapy for newly-diagnosed glioblastoma multiforme. Anticancer Res 2015;35:1229-35 Medline
20. Essig M, Shiroishi MS, Nguyen TB, et al. Perfusion MRI: the five most frequently asked technical questions. $A J R A m$ J Roentgenol 2013;200:24-34 CrossRef Medline

21. Jahng GH, Li KL, Ostergaard L, et al. Perfusion magnetic resonance imaging: a comprehensive update on principles and techniques. Korean J Radiol 2014;15:554-77 CrossRef Medline

22. Stadlbauer A, Pichler P, Karl M, et al. Quantification of serial changes in cerebral blood volume and metabolism in patients with recurrent glioblastoma undergoing antiangiogenic therapy. Eur J Radiol 2015;84:1128-36 CrossRef Medline

23. de Groot JF, Fuller G, Kumar AJ, et al. Tumor invasion after treatment of glioblastoma with bevacizumab: radiographic and pathologic correlation in humans and mice. Neuro Oncol 2010;12:233-42 CrossRef Medline

24. Iwamoto FM, Abrey LE, Beal K, et al. Patterns of relapse and prognosis after bevacizumab failure in recurrent glioblastoma. Neurology 2009;73:1200-06 CrossRef Medline

25. Keunen O, Johansson M, Oudin A, et al. Anti-VEGF treatment reduces blood supply and increases tumor cell invasion in glioblastoma. Proc Natl Acad Sci U S A 2011;108:3749-54 CrossRef Medline 